\title{
Percolation model for electrical breakdown in insulating polymers
}

\author{
Kai $W^{a)}$ and L. A. Dissado \\ Department of Engineering, University of Leicester, Leicester LE1 7RH, United Kingdom \\ Tatsuki Okamoto \\ Central Research Institute of Electric Power Industry, 2-6-1 Nagasaka, Yokosuka 240-0196, Japan
}

(Received 18 March 2004; accepted 29 September 2004)

\begin{abstract}
It is suggested that an electric field above a given value eliminates the barriers to the transport of trapped charge carriers so as to produce an extended state in the form of a percolation cluster, and that the consequent current multiplication results in electrical breakdown. This model provides an estimated value of intrinsic breakdown strength close to the actual value. By considering the interactions between trap barrier potentials, the effect of electrical aging can be explained in terms of an increase in trap density. Many phenomena, such as the effect of weak points and the change of breakdown strength with the content of co-monomers or additives, can also be explained using this model. (C) 2004 American Institute of Physics. [DOI: 10.1063/1.1819526]
\end{abstract}

The mechanism of intrinsic breakdown associated with the transition of electron behavior at high electrical field in insulating polymers is still not clearly understood. Their conduction band is near or above the vacuum level, a large energy gap $(\sim 8 \mathrm{eV})$ exists between the valence and the conduction bands, ${ }^{1}$ and the mean free path is less than $3 \mathrm{~nm} .{ }^{2,3}$ These factors cause the breakdown strength calculated from the classical models for electron driven breakdown to be above $10^{9} \mathrm{~V} / \mathrm{m},{ }^{2}$ much larger than experimental values of the order of $10^{8} \mathrm{~V} / \mathrm{m}$. It is usually argued that this reduction is related to weak points, such as free volume, submicrovoids, and low-density regions. In the model of free-volume breakdown $^{4,5}$ it is noted that the largest empty spaces in polymers may be as large as several decades of nanometers, ${ }^{5}$ the longest free path of electron can therefore be large enough for electrons to overcome the trap barrier or to induce impact ionization. However, this model seems only able to describe the development of degradation around the large free volume regions, it is unsatisfactory to explain the overall current multiplication in an intrinsic breakdown process. A contrary example is partial discharge in which breakdown may not occur at all even though the internal gas cavity has been discharged.

In respect of electrical aging it has been suggested that the creation of low-density regions near the electrodes at high fields is a necessary step leading to breakdown. ${ }^{6,7}$ The formation of such regions is associated with molecular dissociation due to the energy released in the trapping process of hot electrons, ${ }^{2}$ in kinetic energy transfer during spacecharge injection and extraction processes, ${ }^{8}$ or lattice deformation on charge trapping. ${ }^{9}$ However, these aging models do not seem suitable for instantaneous impulse breakdown when low-density regions have no time to form. It is also still not very clear how the molecular dissociation affects the electron behavior and the breakdown strength. Other models, such as the filamentary theories ${ }^{10,11}$ for the creation of a breakdown path and the simulation models ${ }^{12,13}$ for the shape of breakdown paths, use the breakdown strength as a parameter. They

\footnotetext{
${ }^{a)}$ Also at: Central Research Institute of Electric Power Industry, 2-6-1 Nagasaka, Yokosuka 240-0196, Japan; electronic mail: wukai@criepi.denken.or.jp
}

are not concerned with the physics behind the transition of the electron state in the breakdown process. This letter proposes a percolation model for intrinsic breakdown, which not only provides estimated breakdown strengths close to the actual value, but also describes the ageing effect on breakdown strength.

Because the band gap is so large $(>8 \mathrm{eV})$ in insulating polymers very few charge carriers are produced by thermal promotion from the valence band to the conduction band. Instead the conductivity is mainly due to charges produced by ionized donor (acceptor) molecules and injected charges, which hop between localized states (or traps).

Ionized donors (acceptors) produce traps of the PooleFrenkel type in which the barrier potential energy function is assumed to be of Coulombic form following a simple inverse square law with distance from the site. In the presence of a field $E$, the trap barrier is reduced by $\Delta \varphi .^{14}$

$$
\Delta \varphi=\left(e^{3} / \pi \varepsilon\right)^{1 / 2} E^{1 / 2},
$$

where $\varepsilon$ is the dielectric permittivity.

Equation (1) implies that a trap barrier may decrease to zero when the field becomes high enough. In polymers, the traps are distributed over a range of trap depths, with consequently a range of fields required to eliminate them as traps. Clearly, if the field is so high that all barriers are reduced to zero, extended states will be formed and the charge carriers can move freely over all space. This will result in an abrupt increase in charge mobility and electron mean free path, and an irreversible breakdown via current multiplication and impact ionization becomes possible. This is the condition for breakdown in one-dimensional systems. However, in two or three dimensions, according to percolation theory, ${ }^{15,16}$ an infinite percolation path (i.e., the extended state) may be formed when the barriers of only a fraction of the traps disappear.

We have represented the polymer by a three-dimensional cubic network (Fig. 1), in which each site stands for a trap with its barrier uniformly distributed in a range from 0 to $1 \mathrm{eV}$. Each site has 26 neighbor sites and thus 26 bonds connected to it. When the field is zero, all the bonds are nonconductive. Assuming the distance between traps is large enough that the interactions between the Coulombic 


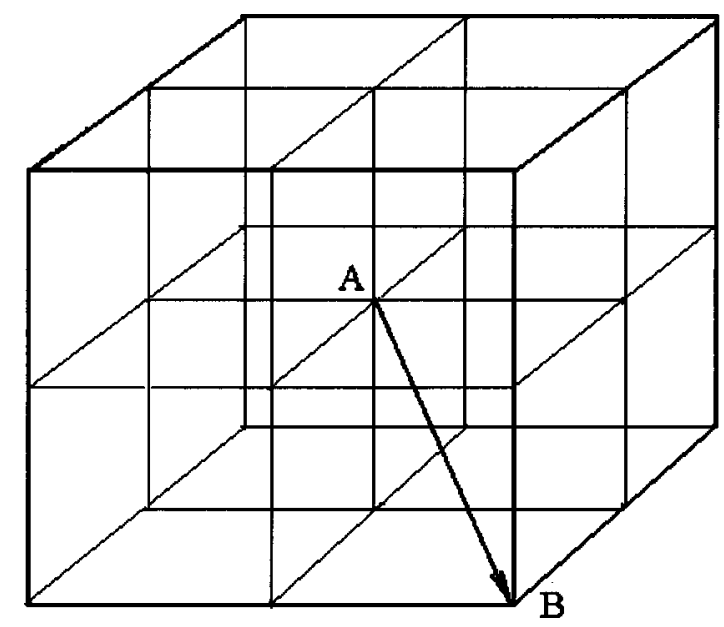

FIG. 1. A sketch for a three-dimensional cubic network.

site potentials can be neglected, the bond $\mathbf{A B}$ from site $A$ to site $B$ becomes conductive when the following condition is satisfied:

$$
E \cos \theta>\pi \varepsilon \varphi_{A}^{2} / e^{3},
$$

where $\varphi_{A}$ is the barrier at site $\mathrm{A}$, and $\theta$ is the angle between the field $E$ and the bond direction vector $\mathbf{B A}$.

In an infinite system, the conductive bonds can connect to form an infinite conductive path (i.e., the percolation path) when the field is high enough. By using Monte Carlo simulation, the fraction of the samples with percolation paths can be calculated as a function of a field-dependent parameter $\varphi_{E}$, which is the largest barrier that can be reduced to zero by the field $E$,

$$
\varphi_{E}=\left(e^{3} / \pi \varepsilon\right)^{1 / 2} E^{1 / 2} .
$$

Figure 2 indicates that a percolation threshold $\varphi_{E_{C}}$ does exist with an estimated value of $\varphi_{E_{c}} \approx 0.188$.

Generally, if the trap barrier is uniformly distributed in a range of $[a, b]$, the percolation threshold should be

$$
\varphi_{E_{c}}=0.188(b-a)+a \text {. }
$$

Above the percolation threshold, the percolation probability $P\left(\varphi_{E}\right)$ (i.e., the ratio of the number of bonds in the percolation path to the total number) increases with the field (or $\varphi_{E}$ ), acting as a scaling factor. ${ }^{15,16}$ Thus the number of carriers in the percolation path and the current increase rapidly with the field. Moreover, the increase in $P\left(\varphi_{E}\right)$ also leads to an increase in electron free mean path. When $\varphi_{E}$ just reaches the threshold $\varphi_{E_{c}}$, only a small number of sites are

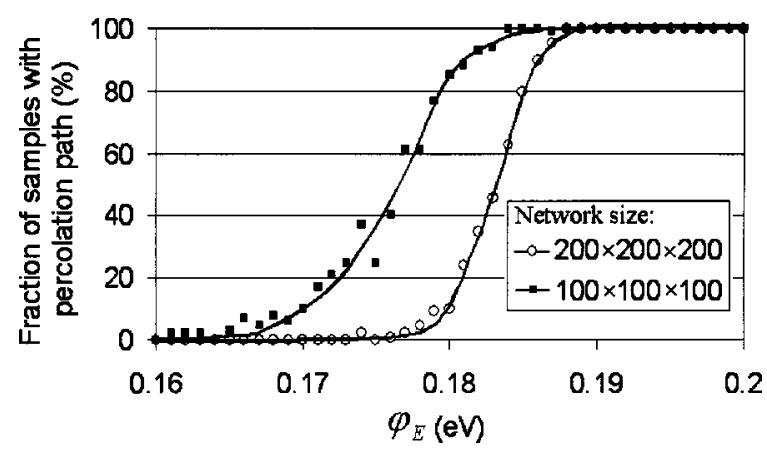

FIG. 2. Variation of the fraction of percolation samples with $\Phi_{E}$.

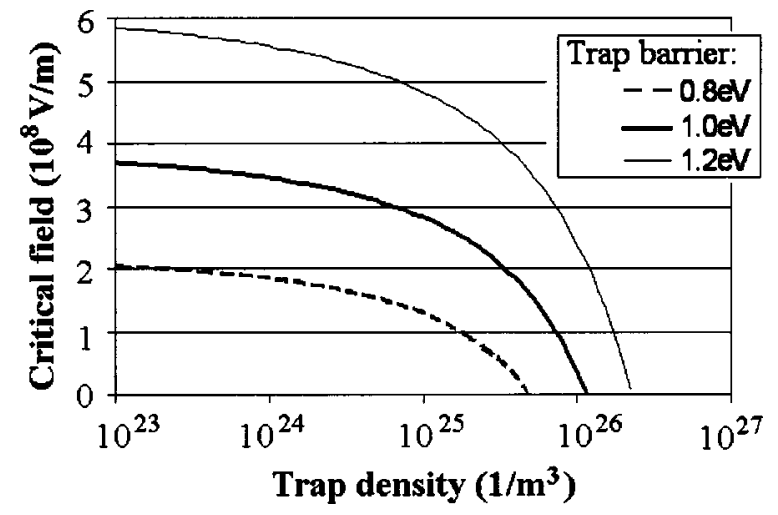

FIG. 3. Relationship between trap density and the critical field for barrier elimination.

included in the percolation path, and carrier movement in the percolation path is dominated by the large number of singly connected sites. This restricts the mean free path, because of the scattering effects of the traps surrounding the percolation path. However, when the percolation probability $P\left(\varphi_{E}\right)$ becomes large at higher field, there are more multiply connected sites and less peripheral sites giving an increase in mean free path. When the carrier free mean path becomes large enough irreversible breakdown may be induced by current multiplication or impact ionization.

Although the breakdown strength is larger than the critical field $\left(E_{c}\right)$ for the extended state, we suppose that it cannot be more than several times of $E_{c}$. In polymers, the traps are mainly distributed in a range of $0.6-1.2 \mathrm{eV} .{ }^{17-19}$ The effect of deeper traps up to $2 \mathrm{eV}$ or even larger may be neglected due to their low density. Shallow traps (or localized states) below $0.5 \mathrm{eV}$ will be present but are difficult to detect though they may be of a high density. If we assume that it is the deeper traps that determine the carrier mobility, and hence that traps in the range of $0.6-1.2 \mathrm{eV}$ play the important role in determining the breakdown strength, the critical field for extended state formation can be estimated to be $2.1 \times 10^{8} \mathrm{~V} / \mathrm{m}$ [referring to Eq. (4)]. This value coincides with the actual breakdown strength of polymers.

If the trap density is high, the trap distance becomes small, and the interaction between the barrier potentials cannot be neglected. Considering the interactions between the barrier potentials of two Poole-Frenkel-type traps, the correlation between the trap density and the critical field for barrier elimination is shown in Fig. 3. It can be deduced that the critical field for percolation threshold decreases with the increase of trap density.

Although we have concentrated on donor traps a similar argument would apply to acceptor (neutral when empty) traps. In this case $\Delta \varphi$ would have a different functional dependence upon $E$ and the numerical values for percolation threshold would be different to those given above, but not by more than a small multiplicative factor. It is not clear whether or not a high acceptor density will increase the probability of percolation path formation since this will depend upon the details of the local potential surface. When both donor and acceptor traps co-exist however, an increase in the density of either will promote percolation path formation.

It has been frequently observed that the breakdown strength of co-polymers at first increased with the comonomer content and then decreased at higher 
concentration. $^{20,21}$ The same behavior was found with additive concentration. ${ }^{22}$ The increase of breakdown strength was explained in terms of the scattering or trapping effect of the co-monomers or additives, which prevented electron acceleration. $^{21,22}$ However, the decrease of breakdown strength at high concentrations is not yet understood. Our percolation model can provide a simple explanation for this kind of phenomena. Co-monomers or additives can introduce new traps with higher barriers, which can be expected to lead to a higher critical field for percolation path formation and thus an increase in breakdown strength. However, when the density of the co-monomers or additives becomes high enough, the interaction between trap barrier potentials causes the reduction of the breakdown strength. It was reported that the maximum breakdown strength was achieved when the content of the co-monomers or additives was at $0.1 \mathrm{~mol} \%$, $0.2-0.3 \mathrm{wt} \%$ and $1.5-6 \mathrm{wt} \%$, respectively. ${ }^{20-22}$ These correspond to an estimated range of $10^{24}-10^{26} \mathrm{~m}^{-3}$ for the molecule density of co-monomers or additives. This range agrees with Fig. 3, which indicates that the critical field $E_{c}$ begins to decrease rapidly when the trap density becomes larger than $10^{24}-10^{25} \mathrm{~m}^{-3}$. The different contents of co-monomers or additives at the breakdown strength peak may be due to different barriers for the traps that are introduced into the polymers.

The enhancement of percolation path formation by the increase in trap density in polymers is similar to the insulator-metal transition in phosphorus-doped silicon when the donor density is increased to a threshold. ${ }^{23}$ This transition is explained as the formation of a percolation path due to the overlapping of donor wave functions, ${ }^{16}$ and can be achieved without an electric field because the Bohr radii of the donor wave function can be much larger than the crystal constant in semiconductors. In polymers, the intrinsic disorder in molecule structure produces intrinsic localized states (or traps) regardless of the content of co-monomers or additives. Therefore, a high electrical field is necessary to reduce the barrier of the intrinsic traps and form the percolation path. This is also different from the percolation-path formation in the case when conducting particles (e.g., carbon black, etc.) are added to polymers. Moreover, practically, unlike Fig. 3, the critical field in copolymers cannot be reduced to zero even when the content of co-monomers is very high.

Figure 3 can be also used to qualitatively describe the ageing effect. In electrical ageing, molecular dissociation, bond scission, or lattice deformation is assumed to occur as the result of a variety of processes, ${ }^{2,8,9}$ all of which can lead to an increase in trap density. In particular molecular dissociation or bond scission may lead to the creation of ions and free radicals, i.e., donor traps. Referring to Fig. 3, final breakdown can be induced when the trap density becomes high enough. This agrees with the assumption that breakdown occurs when the density of free radicals reaches a certain value. ${ }^{24}$ Our model implies that the threshold trap den- sity for percolation path formation should be different at different electrical fields.

The percolation model can also easily explain the fact that breakdown paths tend to pass through weak points (such as cavities and other impurities). In the case of cavities (or nano-holes), the large electron free mean path in these regions may lead to impact ionization and carrier multiplication, so the current passing through these regions in the prebreakdown process tends to be higher. Thus the final breakdown is likely to go through these regions. In the case of impurities that can cause field distortion and local field enhancement, the barriers of surrounding traps may be reduced to zero in advance of the final breakdown due to the high local field. Therefore, the percolation path also tends to pass through these regions. The existence of weak points therefore favors the final breakdown process with their involvement depending statistically upon their density.

This work was supported by EPSRC Grant No. GR/ R90536/01 for K.W.

${ }^{1}$ H. J. Wintle, IEEE Trans. Electr. Insul. 12, 97 (1977).

${ }^{2}$ K. C. Kao, J. Appl. Phys. 55, 752 (1984).

${ }^{3}$ Y. Tanaka, N. Ohnuma, K. Katsunami, and Y. Okhi, IEEE Trans. Electr. Insul. 26, 258 (1991).

${ }^{4}$ J. Artbauer, J. Phys. D 29, 446 (1996).

${ }^{5}$ H. Zhang, H. K. Xie, and Z. Y. Liu, J. Xi'an Jiaotong Univ. 26, 115 (1992) [in Chinese].

${ }^{6}$ E. O. Foster, IEEE Trans. Electr. Insul. 17, 517 (1982).

${ }^{7}$ P. P. Budenstein, IEEE Trans. Electr. Insul. 15, 225 (1980).

${ }^{8}$ T. Tanaka and A. Greenwood, IEEE Trans. Power Appar. Syst. 97, 1749 (1978).

${ }^{9}$ L. Dissado, G. Mazzanti, and G. C. Montanari, IEEE Trans. Dielectr. Electr. Insul. 2, 1147 (1995).

${ }^{10}$ H. R. Zeller and W. R. Schneider, J. Appl. Phys. 56, 455 (1984).

${ }^{11}$ J. C. Fothergill, IEEE Trans. Electr. Insul. 26, 1124 (1991).

${ }^{12}$ L. Niemeyer, L. Pietronero, and H. J. Wiesmann, Phys. Rev. Lett. 52, 1033 (1984).

${ }^{13}$ H. J. Wiesmann and H. R. Zeller, J. Appl. Phys. 60, 1770 (1986).

${ }^{14}$ T. J. Lewis, in Space Charge in Solid Dielectrics, edited by J. C. Fothergill and L. A. Dissado (The Dielectrics Society, 1998), pp. 1-17.

${ }^{15}$ D. Stauffer and A. Aharony, Introduction to Percolation Theory, rev. 2nd ed. (Burgess Science, 1994).

${ }^{16} \mathrm{R}$. Zallen, The Physics of Amorphous Solids (Wiley-Interscience, New York, 1983).

${ }^{17}$ P. K. Watson, IEEE Trans. Electr. Insul. 22, 129 (1987).

${ }^{18}$ G. Mazzanti, G. C. Montanari, and J. M. Alison, IEEE Trans. Dielectr. Electr. Insul. 10, 187 (2003).

${ }^{19}$ L. A. Dissado, O. Paris, T. Ditchi, C. Alquie, and J. Lewiner, Annu. Rep. CEIDP (IEEE Pub. No. 99CH36319), 1999, p. 23.

${ }^{20}$ M. Hikita, I. Kanno, and M. Ieda, IEEE Trans. Electr. Insul. 22, 175 (1987).

${ }^{21}$ Y. Tanaka, Y. Ohki, and M. Ikeda, IEEE Trans. Electr. Insul. 27, 432 (1992).

${ }^{22}$ L. Y. Gao, W. Y. Guo, and D. M. Tu, IEEE Trans. Dielectr. Electr. Insul. 10, 233 (2003).

${ }^{23}$ H. F. Hess, K. DeConde, T. F. Rosenbaum, and G. A. Thomas, Phys. Rev. B 25, 5578 (1982).

${ }^{24}$ D. M. Tu and X. S. Wang, J. Xi'an Jiaotong Univ. 27, 33 (1993) [in Chinese]. 